\title{
iRGD as a tumor-penetrating peptide for cancer therapy (Review)
}

\author{
HONG YIN $^{1,2^{*}}$, JIE YANG $^{1 *}$, QING ZHANG $^{1}$, JIE YANG $^{1,2}$, HAIYU WANG $^{1}$, \\ JINJING XU ${ }^{1}$ and JUNNIAN ZHENG ${ }^{1,3,4}$ \\ ${ }^{1}$ Cancer Institute, Xuzhou Medical University, Xuzhou, Jiangsu 221002; \\ ${ }^{2}$ Center of Radiotherapy of The Second Affiliated Hospital of Xuzhou Medical University, \\ Xuzhou, Jiangsu 221006; ${ }^{3}$ The Cancer Center of The Affiliated Hospital of Xuzhou Medical University; \\ ${ }^{4}$ Jiangsu Center for The Collaboration and Innovation of Cancer Biotherapy, Cancer Institute, \\ Xuzhou Medical University, Xuzhou, Jiangsu 221002, P.R. China
}

Received January 27, 2016; Accepted January 23, 2017

DOI: $10.3892 / \mathrm{mmr} .2017 .6419$

\begin{abstract}
As a tumor-targeting and -penetrating peptide, iRGD binds to $\alpha \mathrm{v}$ integrins and neuropilin- 1 receptors, which are expressed at high levels on tumor cells and the surfaces of vasculature. Subsequently, iRGD penetrates deep into the tumor parenchyma with antitumor drugs, imaging agents, immune modulators and biological products. These substances are either chemically linked to the peptide or co-injected with the peptide. The iRGD peptide can be readily synthesized, exhibits significantly improved penetration, compared with traditional peptides, and can effectively inhibit tumor metastasis. Therefore, the peptide is now used widely for the diagnosis and treatment of cancer. However, whether the peptide is able to promote the entry of drugs into non-targeted cells remains to be fully elucidated. In this review, an overview of iRGD is presented, focusing on its identification, mechanism of action and previous studies on its roles in various types of cancer. Studies in previous years have demonstrated the potential of the iRGD protein for tumors diagnosis and targeted treatment, which warrants further investigation.
\end{abstract}

\section{Contents}

1. Introduction

2. Initial peptide identification

3. Mechanism of iRGD penetration

4. iRGD for tumor diagnosis and treatment

5. Conclusion

Correspondence to: Professor Junnian Zheng, Cancer Institute, Xuzhou Medical University, 84 West Huai-hai Road, Xuzhou, Jiangsu 221002, P.R. China

E-mail: jnzheng@xzmc.edu.cn

*Contributed equally

Key words: penetrating peptide, mechanism, application, cancer treatment, diagnosis

\section{Introduction}

Cancer is a global health problem, and is gradually becoming the primary contributor to mortality rates in women and men in developing and developed countries (1). In previous years, chemotherapy, radiotherapy, surgery and immunotherapy have been the primary treatment methods for cancer (2). Chemotherapy has always been considered the principle and most common method, however, it exhibits drawbacks, including the low concentration of chemotherapeutic drug at the tumor site, and serious side effects due to chemotherapeutic drugs not specifically targeting tumors and always affecting normal cells/tissues. Furthermore, chemotherapeutic drugs only possess a limited capacity to penetrate into the parenchyma of solid tumors. Due to these limitations, improving the efficiency of chemotherapeutic drugs is an urgent requirement $(3,4)$.

As drugs for the treatment of solid tumors can only penetrate 3-5 cell diameters from blood vessels (5), the low concentration of anticancer drugs at the tumor site is a substantial obstacle for tumor treatment, which limits the anticancer efficacy and suggests that the effective drug concentration at the tumor site is markedly lower, compared with the dose of exposure. In previous decades, cancer studies have focused on tumor-targeting peptides, which support drug penetration. Conventional RGD-peptides bind selectively to integrins $\alpha v \beta 3$ and $\alpha v \beta 5$, which are excessively expressed on several types of tumor (6). These peptides successfully deliver drugs, nanoparticles, viruses and biologicals to the blood vessels (7), and marginally increase accumulation within the tumor, however, their limited loading capacity in the tumor parenchyma remains a challenge for cancer therapy.

In previous years, improved novel peptides aimed at identifying tumor blood vessels and tumor cells appear to be more effective in the treatment of tumors, compared with traditional penetrating peptides $(7,8)$. The iRGD (CRGDKGPDC) peptide has been developed on the basis of RGD peptides and is composed of nine amino acids. It first binds to $\alpha v$ integrins, which are expressed on tumor cells and vessels. Subsequently, it is cleaved by proteases to expose the neuropilin-1 (NRP-1)-binding CRGDK/R, which effectively 
triggers the tumor penetration process $(7,9)$. The peptide is used to increase the pore diameter and surface area of tumor blood vessels, and to reduce the pressure effect in the tumor interstitium, increasing the rate of diffusion of small molecule drugs (10-12). The scientific interest in iRGD has resulted from its binding to NRP-1 in particular, as this triggers extravasation (13). Furthermore, iRGD specifically penetrates into angiogenic vessels and tumor tissues. Due to this novel delivery system and the low toxicity to normal cells, iRGD has attracted significant attention (14). Further advantages of iRGD arise from its simple and low-cost synthesis. Coupled with iRGD, drugs, nanoparticles and proteins can be effectively delivered to the tumor site, which reduces side effects. At present, the iRGD peptide is widely used in the diagnosis and treatment of tumors.

\section{Initial peptide identification}

The tumor vasculature is indispensable in the process of tumor growth and metastasis, as it not only provides the tumor with the necessary nutrients and oxygen, but also assists in the transportation of cancer cells to adjacent or distant organs for tumor metastasis (15). Therefore, the tumor vasculature can be considered as the target of tumor diagnosis and treatment. Phage peptides, first identified by Pasqualini and Ruoslahti in 1996, have been widely used (16). The homing peptide on the phage coat protein combines with target molecules in vascular endothelial cells. A mass screening of the phage allows for the selective detection of targeted phage peptides, which combine into tumor blood vessels (17-19). Using this method, an increasing number of peptides targeting the tumor vasculature can be identified. Substantial experimental data have shown that these peptides can deliver drugs to tumors and act as effective image contrast agents $(20,21)$, thus are important in tumor diagnosis and treatment. RGD and NGR are the two most well-known short peptides targeting tumor vasculature in phages. iRGD has been developed as a novel type of tumor-penetrating peptide, which not only targets tumor blood vessels, but can also deliver drugs deep into the tumors. The iRGD peptide results from the connecting of RGD with NRP-1 peptide ligands. iNGR was designed based on known sequence elements (22).

\section{Mechanism of iRGD penetration}

The RGD sequence (Arg-Gly-Asp) selectively binds to $\alpha v \beta 3$ and $\alpha v \beta 5$ integrins which are overexpressed in the tumor vasculature (23). Further studies have shown that connecting the peptide ligands of NRP-1 and RGD improves drug delivery to the extravascular tissues in addition to the tumor blood vessels. The target peptide CendR, can only be activated when the C-terminus of the motif is exposed. Subsequently, the activated CendR can bind to NRP-1, which is expressed at high levels on tumor tissues, to initiate deep tissue penetration (8). In general, iRGD acts through the following steps: RGD peptides bind to $\alpha v \beta 3$ and $\alpha v \beta 5$ integrins on tumor cells and tumor blood vessel endothelial cells. Secondly, under the action of cellular proteases, the peptide is cleaved to expose the activated CendR motif at the C-terminus. Finally, CendR binds to NRP-1, which triggers tumor tissue penetration carrying the cargo at the N-terminus(Fig. 1).

\section{4. iRGD for tumor diagnosis and treatment}

$i R G D$ in tumor preclinical diagnosis. In 2009, Sugahara et al intravenously infused iRGD-linked iron oxide nanoworms into tumor-burdened mice. A low MRI signal intensity was detected in the entire tumor, which was confirmed using MRI imaging. Therefore, the detection methods consistently indicated the superiority of iRGD over the RGD peptide in terms of its ability to transfer diagnostic agents to tumors (7). In 2011, Ye et al synthesized the two near-infrared fluorescence-labeled iRGD peptides, Ac-Cys (IRDye800CW)-iRGD and DOTA-Cys (IRDye800CW)-iRGD. These peptides were injected intravenously into mice bearing MDA-MB-435 tumors, which revealed the tumor locations (24). In 2015, iRGD-modified porous silicon nanoparticles were verified for cancer theranostics through in vitro and in vivo experiments (25). This revealed the potential of the iRGD peptide as a diagnostic reagent in clinical practice.

iRGD mediates drug delivery for cancer treatment. The mode of action of iRGD is well understood and its preclinical application is developing widely. Substantial investigations have been performed to investigate the application of iRGD as an antitumor agent in vivo and in vitro, which are briefly summarized in the following.

Sugahara et al (26) reported that co-injection with iRGD enhances the antitumor effect of free drugs without a chemical entity, and reduced the side effects of drugs in mice bearing five tumors, including human breast cancer, prostate cancer and pancreatic adenocarcinoma. Investigations have revealed the simplicity and effectiveness of the co-injection of iRGD and tumor-targeting drugs. This combination does not alter the chemical structure of the drugs, thus avoiding structural alterations, which may reduce the activity of the functional drugs. These findings also encourage the co-administration of iRGD in various forms of drug applications for future investigations in the treatment of different types of tumor. Sugahara et al also reported that iRGD effectively improved the curative effect of doxorubicin (DOX) in inhibiting peritoneal carcinomatosis. It was confirmed that the intratumoral aggregation of intraperitoneally co-injected DOX in mice was $\sim 1.5$ times higher. Based on this finding, it was concluded that intraperitoneally co-administered iRGD can be regarded as a simple and effective way to inhibit the progression of peritoneal carcinomatosis and improve the effect of chemotherapy (26).

In studies involving transplantation models, cisplatin (CDDP) was co-administered with iRGD resulting in an increase in survival rates by $30 \%$ and a substantial reduction in the toxicity of the chemotherapeutic drugs (27).

In 2013, Gu et al associated iRGD with paclitaxel-loaded nanoparticles for drug delivery to the C6 glioma parenchyma. Mice bearing C6 glioma cells, which were treated with the iRGD-associated nanoparticles, had markedly increased survival rates, compared with the mice, which had not received the iRGD-associated treatment (28).

iRGD has also been selected as a targeting ligand and has been used for the modification of sterically-stabilized 


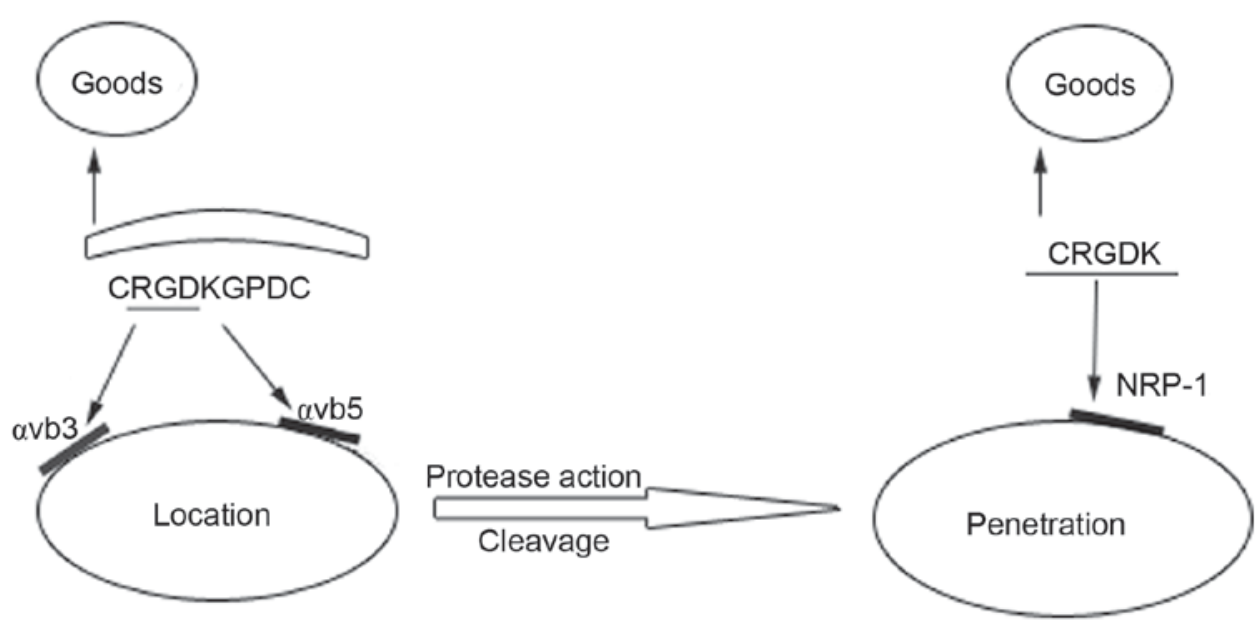

Figure 1. Mechanism of iRGD in tumor penetration. The first step is termed 'location', RGD binds to $\alpha v \beta 3$ and $\alpha v \beta 5$ integrins, which are expressed at high levels on tumor cells and tumor blood vessel endothelial cells. The circular peptide is then cleaved to the peptide CRGDK by the protease. In the final step, 'penetration', CRGDK binds to NRP-1 receptor to penetrate deep into the tumor substance, including the load at the C-terminal end. NRP-1, neuropilin-1.

liposomes (SSLs). Chemotherapeutic drugs (CLA-PTXorDOX) were loaded to these liposomes to yield iRGD-SSL-CLA-PTX or iRGD-SSL-DOX, which were assessed in C57BL/6 mice bearing B16-F10 tumors, respectively. The results showed that the tumor volumes were significantly reduced upon iRGD-SSL-CLA-PTX or iRGD-SSL-DOX treatment, compared with tumors in mice, which had not received the corresponding iRGD modification $(29,30)$.

For the efficient delivery of the DOX-polymer to tumor tissues, Wang et al synthesized an iRGD-PPCD conjugate, which was injected intravenously into mice with subcutaneously implanted C6-glioma-tumors. These in vivo investigations showed that iRGD-PPCD has a superior penetrating capacity, compared with RGD. Statistical analysis revealed that the median duration of survival of the mice following treatment with iRGD-mediated drugs was longer, compared with that following treatment with RGD-mediated drugs, indicating that iRGD improved the antitumor effect and penetration efficacy (31).

Akashi et al developed pancreatic cancer models. They treated tumor-burdened nude mice with a combination of gemcitabine (GEM) and iRGD, and observed prominent tumor reduction, compared with mice treated with GEM only in the cell line-based xenografts (32).

Derived from the anti-apoptotic protein, Bfl-1, amphipathic tail-anchoring peptide (ATAP) is used as a mitochondrial targeting peptide. The ATAP modifications, ATAP-iRGD-M8 and ATAP-iRGD exhibit improved stability and solubility, and improved capacity in selective delivery to tumor tissues. The two peptides significantly decreased tumor sizes in nude mice burdened with DU145 and PC3 cells (14).

Puig-Saus et al (33) inserted iRGD into an oncolytic adenovirus to increase adenovirus penetration to the tumor mass. By inserting the peptide, nude mice bearing subcutaneous A549 and MIA PaCa-2 xenograft tumors showed improved tumor growth control, compared with mice without iRGD, and extended mean survival rates. These findings effectively demonstrated that the iRGD peptide enhances transduction, intratumoral dissemination and adenovirus infiltration into the tumor parenchyma to exert an antineoplastic effect $(26,33)$.
Porous silicon (PSi) has been coupled with iRGD as a drug delivery carrier. The final sorafenib-loaded PSi-iRGD has shown a more marked antitumor effect in vitro, which again confirmed the above-described effect of iRGD (34).

Epidermal growth factor receptors (EGFRs), which are closely associated with the prognosis of cancer, are expressed at high levels on the surfaces of different human tumor cells, including gastric cancer and gastric lung cancer. It has been reported that anti-EGFR-iRGD, a composite protein targeting $\alpha v \beta 3, \alpha v \beta 5$, NRP-1 and EGFRs, shows potent tumor tissue penetration ability. In vivo investigations of mice bearing subcutaneous BGC-823, which received intraperitoneal injections, showed that the combination of DOX with anti-EGFR-iRGD was more effective at inhibiting BGC-823 MCS growth, compared with chemotherapeutic drugs (35).

A report in 2015 described the co-administration of DOX-loaded, CDDP-crosslinked and polysaccharide-based nanoparticles (Dex-SA-DOX-CDDP) with iRGD, afford marked advantages in inhibiting the tumor growth and metastasis of murine colorectal carcinoma and metastatic mammary carcinoma, compared with the corresponding nanoparticles without iRGD co-administration (36). Peng and Kopeček conjugated matrix metalloproteinase-2 with iRGD, and the resulting novel tumor-penetrating peptide conjugates exhibited the highest cytotoxicity towards DU-145 cells, which prompted further investigation (37). Zhang et al established a human non-small cell lung cancer xenograft nude mice model with A549 cells. Treatment comprising a combination of GEM and iRGD was applied, which inhibited the growth of tumors, which were not sensitive to the same dose of GEM alone. Apoptotic cells in the tumor tissues were detected using a TUNEL assay and the statistical analysis revealed that the highest apoptotic index was for the groups treated with the GEM/iRGD combination (38).

Wang et al demonstrated that iRGD-modified nanoparticles improved the tumor absorption of nanoparticles when injected intravenously. Intratumor injection confined the nanoparticles to the tumor to a greater degree, compared with the free drug (25). This study revealed another application of the iRGD peptide. 
Table I. iRGD as a carrier applied in tumor treatment.

\begin{tabular}{|c|c|c|c|}
\hline Author, year & Load carried by iRGD & Tumor & (Refs.) \\
\hline & Drug & & \\
\hline Sugahara et al, 2010 & DOX & Peritoneal carcinomatosis & (26) \\
\hline Song et al, 2012 & CDDP & A549 & (27) \\
\hline Du et al, 2014 & iRGD-SSL-CLA-PTX & B16-F10 & (29) \\
\hline Yu et al, 2013 & iRGD-SSL-DOX & B16-F10 & $(30)$ \\
\hline Wang et al, 2014 & Paclitaxel, iRGD-PPCD & C6 glioma & $(31)$ \\
\hline De et al, 2014 & ATAP-iRGD-M8 & DU145 and PC3 & (14) \\
\hline $\begin{array}{l}\text { Sugahara et al, 2010; } \\
\text { Puig-Saus et al, } 2014\end{array}$ & Oncolytic adenovirus & A549 and MIA PaCa-2 & $(26,33)$ \\
\hline Sha et al, 2015 & DOX-anti-EGFRs & BGC-823 & (35) \\
\hline Li et al, 2015 & Dex-SA-DOX-CDDP & Colorectal carcinoma & (36) \\
\hline \multirow[t]{2}{*}{ Zhang et al, 2015} & Gemcitabine & A549 & (38) \\
\hline & Biological product & & \\
\hline Lao et al, 2014 & Thymopoietin pentapeptide & Melanoma & (39) \\
\hline Chen et al, 2013 & Cell death domain & MCF-10CA1a & $(40)$ \\
\hline Mao et al, 2015 & M-SAL & Cancer stem cells & (41) \\
\hline
\end{tabular}

DOX, doxorubicin; CDDP, cisplatin; PPCD, PEGylated-polyamidoamine-cis-aconityl-DOX; SSL, sterically stabilized liposome; ATAP, anti-apoptotic peptide; EGFRs, epidermal growth factor receptors; M-SAL, DSPE-PEG (2000) nanomicelles-salinomycin.

iRGD mediates biological product delivery for cancer treatment. In addition to chemotherapeutic drugs, Lao et al introduced the iRGD peptide sequence into the C-terminus of the thymopoietin pentapeptide, TP5, to improve the poor penetration ability of TP5. The findings suggested that the injections of TP5-iRGD inhibited melanoma progression more effectively, compared with the native peptide. In addition, computational observations of the mechanism of activity confirmed the potential of the peptide for tumor therapy (39).

Chen et al reported that the cell death domain (CDD) was effective for inducing cell apoptosis, therefore, CDD was fused to iRGD to obtain a tissue-penetrating protein. By injecting the protein intratumorally into mice bearing orthotopically-implanted MCF-10CA1a breast tumors, CDD-iRGD inhibited the growth of tumors, with a decrease in the tumor volumes by $77 \%$ (40). These studies reflect potential applications of iRGD coupled with biological products. The conjugation of iRGD with DSPE-PEG (2000) nanomicelles-salinomycin (M-SAL) to yield M-SAL-iRGD also exhibited a prominent increase in cytotoxicity in cancer stem cells and liver cancer cells (41) (Table I).

iRGD inhibits metastasis. Metastasis is the primary cause of cancer-associated mortality, and studies have focused increasingly on the development of methods for improving resistance and treating cancer metastasis. The iRGD peptide itself does not affect the survival of cells (42), however, it has been reported to inhibit the metastasis of tumors.

As a tissue-penetrating peptide, iRGD efficiently delivers drugs and biological products to various tumors in rodent models. In 2015, Sugahara et al suggested that the iRGD peptide also spontaneously inhibits tumor metastasis, which was confirmed experimentally. Depending on NRP-1, iRGD inhibits spontaneous tumor metastasis, but has no effect on the size of primary tumors. Sugahara et al demonstrated this by developing nude mice models via the orthotopic transplantation of GFP-PC-3 and LM-PmC cells, which led to spontaneous metastases in different organs. The peptide was then injected intravenously into the tumor-bearing mice and circulated for $1 \mathrm{~h}$. Based on the analysis of a series of in vivo and in vitro experiments, it was confirmed that the iRGD peptide inhibited tumor cells migration (42).

In 2015, Hamilton et al reported that iRGD nanoparticles significantly inhibited tumor development as long as they were applied in the early metastatic phase of tumor progression (43). Ni et al confirmed that only decorated nanocrystallites achieved complete intratumoral transfer and accessed cancer stem cells in the murine model, leading to the inhibition of 4T1 proliferation and metastasis (44).

\section{Conclusion}

iRGD consists of two motifs: The RGD motif, which binds to $\alpha \mathrm{v}$ integrins aiming to target drugs accumulated in tumor tissues, and the CendR motif, which binds to NRP-1 to effectively inhibit tumor metastasis. In combination with contrast agents, chemotherapeutic drugs, nanoparticles or proteins, the iRGD peptide can be effectively delivered to the tumor site, reduce the side effects of drugs and improve the curative efficacy of drugs. In previous years, the application of the iRGD peptide for cancer diagnosis and therapy was assessed in pre-clinical in vivo and in vitro experiments, revealing promising results for tumor treatment. In addition to the progress in phage library technology and the development of new screening technologies, the iRGD tumor-tissue-penetrating peptide is likely to be more widely applied in the clinical diagnosis and treatment of tumors. 
It is noteworthy that the iRGD peptide was investigated in a mice model with subcutaneously transplantated tumors, whereas tumor xenograft models did not actually imitate the complex microenvironment of the cancer cells originated from the organ. Therefore, the interpretation of experimental results can have significant limitations. The microenvironment is important for balancing the factors of promoting angiogenesis and anti-angiogenesis, which determine the heterogeneity of angiogenesis in tumors (45). Studies, including a study by Hoffman et al (46-48) independently confirmed that tumor-penetrating peptides are importantinanudemousemodelwithorthotopically-transplanted tumors.

A variety of inflammatory cells, including lymphocytes, neutrophils and macrophages, infiltrate the tumor microenvironment and can secrete different types of cytokines, including growth factors and chemokines (49). Almost all of these inflammatory mediators are involved in tumor angiogenesis. Chronic inflammation is usually accompanied by regeneration and angiogenesis, which may increase the risk of certain types of cancer. Based on reports that tumors and inflammatory tissues have certain biomarkers in common, it is likely that tumor blood vessel-targeted peptides are also combined in the blood vessels of inflammatory diseases, similar to inflammation being associated with the formation of blood vessels. Lahdenranta et al (50) reported that RGD and NGR can be combined into the vessels of hypoxia-induced retinopathy and Buehler et al (51) indicated that the two peptides target the blood vessels of ischemic hearts. These findings further increase the scope of possible applications of the tumor-penetrating peptide, iRGD.

In conclusion, the iRGD peptide interacts with integrin and its ligand, has promising application prospects, and identifies tumors early for diagnosis and treatment. iRGD can be combines with chemical drugs, immune modulators and cytokines to effectively penetrate tumors, and exert more marked antineoplastic effects. The effect of the peptide has been confirmed by a substantial number of animal experiments, confirming significant effects and minimal side effects. Therefore, iRGD offers promise for improving the treatment of tumors in humans. However, for final application in cancer treatment, iRGD requires further investigation and successful outcomes in clinical trials.

\section{Acknowledgements}

This review was supported by grants from the Nature Science Foundation of China (grant no. 81301946), the China Postdoctoral Science Foundation Funded Project (grant nos. 2013M540467 and 53470135), the Nature Science Foundation of Jiangsu Province (grant no. BK2012146) and the Jiangsu Provincial Office of Education Foundation (grant no. JHB2012-34).

\section{References}

1. Rahimi Z, Kasraei R, Najafi F, Tanhapoor M, Abdi H, Rahimi Z, Vaisi-Raygani A, Aznab M and Moradi M: Cancer notification at a referral hospital of Kermanshah, Western Iran (2006-2009). Asian Pac J Cancer Prev 16: 133-137, 2015.

2. Yang J, Zhang Q, Li K, Yin H and Zheng JN: Composite peptide-based vaccines for cancer immunotherapy (Review). Int J Mol Med 35: 17-23, 2015
3. Rabbani-Chadegani A, Paydar P, Amirshenava M and Aramvash A: An in vitro study on the effect of vinca alkaloid, vinorelbine, on chromatin histone, HMGB proteins and induction of apoptosis in mice non-adherent bone marrow cells. Drug Chem Toxicol 38: 220-226, 2015.

4. Hai-Tao Z, Hui-Cheng L, Zheng-Wu L and Chang-Hong G: A tumor-penetrating peptide modification enhances the antitumor activity of endostatin in vivo. Anticancer Drugs 22: 409-415, 2011

5. Minchinton AI and Tannock IF: Drug penetration in solid tumours. Nat Rev Cancer 6: 583-592, 2006.

6. Xiong JP, Stehle T, Diefenbach B, Zhang R, Dunker R, Scott DL, Joachimiak A, Goodman SL and Arnaout MA: Crystal structure of the extracellular segment of integrin alpha Vbeta3. Science 294: 339-345, 2001

7. Sugahara KN, Teesalu T, Karmali PP, Kotamraju VR, Agemy L, Girard OM, Hanahan D, Mattrey RF and Ruoslahti E: Tissue-penetrating delivery of compounds and nanoparticles into tumors. Cancer Cell 16: 510-520, 2009.

8. Teesalu T, Sugahara KN, Kotamraju VR and Ruoslahti E: C-end rule peptides mediate neuropilin-1-dependent cell, vascular, and tissuepenetration.Proc Natl Acad SciUSA 106: 16157-16162,2009.

9. Desgrosellier JS and Cheresh DA: Integrins in cancer: Biological implications and therapeutic opportunities. Nat Rev Cancer 10: 9-22, 2010

10. Emerich DF, Snodgrass P, Dean RL, Lafreniere D, Agostino M, Wiens T, Xiong H, Hasler B, Marsh J, Pink M, et al: Bradykinin modulation of tumor vasculature: I. activation of $\mathrm{B} 2$ receptors increases delivery of chemotherapeutic agents into solid peripheral tumors, enhancing their efficacy. J Pharmacol Exp Ther 296: 623-631, 2001.

11. Emerich DF, Dean RL, Snodgrass P, Lafreniere D, Agostino M, Wiens T, Xiong H, Hasler B, Marsh J, Pink M, et al: Bradykinin modulation of tumor vasculature: II. activation of nitric oxide and phospholipase A2/prostaglandin signaling pathways synergistically modifies vascular physiology and morphology to enhance delivery of chemotherapeutic agents to tumors. J Pharmacol Exp Ther 296: 632-641, 2001.

12. Li CJ, Miyamoto Y, Kojima Y and Maeda H: Augmentation of tumour delivery of macromolecular drugs with reduced bone marrow delivery by elevating blood pressure. Br J Cancer 67: 975-980, 1993.

13. Kadonosono T, Yamano A, Goto T, Tsubaki T, Niibori M, Kuchimaru T and Kizaka-Kondoh S: Cell penetrating peptides improve tumor delivery of cargos through neuropilin-1-dependent extravasation. J Control Release 201: 14-21, 2015.

14. De G, Ko JK, Tan T, Zhu H, Li H and Ma J: Amphipathic tail-anchoring peptide is a promising therapeutic agent for prostate cancer treatment. Oncotarget 5: 7734-7747, 2014.

15. Nyberg P, Salo T and Kalluri R: Tumor microenvironment and angiogenesis. Front Biosci 13: 6537-6553, 2008.

16. Pasqualini R and Ruoslahti E: Organ targeting in vivo using phage display peptide libraries. Nature 380: 364-366, 1996.

17. $\mathrm{Li} \mathrm{ZJ}$ and Cho CH: Development of peptides as potential drugs for cancer therapy. Curr Pharm Des 16: 1180-1189, 2010.

18. Enbäck $J$ and Laakkonen P: Tumour-homing peptides: Tools for targeting, imaging and destruction. Biochem Soc Trans 35: 780-783, 2007.

19. Trepel M, Pasqualini R and Arap W: Chapter 4. Screening phage-display peptide libraries for vascular targeted peptides. Methods Enzymol 445: 83-106, 2008.

20. Ruoslahti E, Bhatia SN and Sailor MJ: Targeting of drugs and nanoparticles to tumors. J Cell Biol 188: 759-768, 2010.

21. Laakkonen $\mathrm{P}$ and Vuorinen K: Homing peptides as targeted delivery vehicles. Integr Biol (Camb) 2: 326-337, 2010.

22. Myrberg H, Zhang L, Mäe M and Langel U: Design of a tumor-homing cell-penetrating peptide. Bioconjug Chem 19: 70-75, 2008.

23. Assa-Munt N, Jia X, Laakkonen P and Ruoslahti E: Solution structures and integrin binding activities of an RGD peptide with two isomers. Biochemistry 40: 2373-2378, 2001.

24. Ye Y, Zhu L, Ma Y, Niu G and Chen X: Synthesis and evaluation of new iRGD peptide analogs for tumor optical imaging. Bioorg Med Chem Lett 21: 1146-1150, 2011

25. Wang CF, Sarparanta MP, Mäkilä EM, Hyvönen ML, Laakkonen PM, Salonen JJ, Hirvonen JT, Airaksinen AJ and Santos HA: Multifunctional porous silicon nanoparticles for cancer theranostics. Biomaterials 48: 108-118, 2015.

26. Sugahara KN, Teesalu T, Karmali PP, Kotamraju VR, Agemy L, Greenwald DR and Ruoslahti E: Coadministration of a tumor-penetrating peptide enhances the efficacy of cancer drugs. Science 328: 1031-1035, 2010. 
27. Song W, Li M, Tang Z, Li Q, Yang Y, Liu H, Duan T, Hong H and Chen X: Methoxypoly(ethylene glycol)-block-poly(L-glutamic acid)-loaded cisplatin and a combination with iRGD for the treatment of non-small-cell lung cancers. Macromol Biosci 12: $1514-1523,2012$

28. Gu G, Gao X, Hu Q, Kang T, Liu Z, Jiang M, Miao D, Song Q, Yao L, Tu Y, et al: The influence of the penetrating peptide iRGD on the effect of paclitaxel-loaded MT1-AF7p-conjugated nanoparticles on glioma cells. Biomaterials 34: 5138-5148, 2013

29. Du R, Zhong T, Zhang WQ, Song P, Song WD, Zhao Y, Wang C, Tang YQ, Zhang X and Zhang Q: Antitumor effect of iRGD-modified liposomes containing conjugated linoleic acid-paclitaxel (CLA-PTX) on B16-F10 melanoma. Int J Nanomedicine 9: 3091-3105, 2014

30. Yu KF, Zhang WQ, Luo LM, Song P, Li D, Du R, Ren W, Huang D, Lu WL, Zhang X and Zhang Q: The antitumor activity of a doxorubicin loaded, iRGD-modified sterically-stabilized liposome on B16-F10 melanoma cells: In vitro and in vivo evaluation. Int J Nanomedicine 8: 2473-2485, 2013.

31. Wang K, Zhang X, Liu Y, Liu C, Jiang B and Jiang Y: Tumor penetrability and anti-angiogenesis using iRGD-mediated delivery of doxorubicin-polymer conjugates. Biomaterials 35: 8735-8747, 2014

32. Akashi Y, Oda T, Ohara Y, Miyamoto R, Kurokawa T, Hashimoto S, Enomoto T, Yamada K, Satake M and Ohkohchi N: Anticancer effects of gemcitabine are enhanced by co-administered $\mathrm{iRGD}$ peptide in murine pancreatic cancer models that overexpressed neuropilin-1. Br J Cancer 110: 1481-1487, 2014.

33. Puig-Saus C, Rojas LA, Laborda E, Figueras A, Alba R, Fillat C and Alemany R: iRGD tumor-penetrating peptide-modified oncolytic adenovirus shows enhanced tumor transduction, intratumoral dissemination and antitumor efficacy. Gene Ther 21: 767-774, 2014

34. Wang CF, Mäkilä EM, Kaasalainen MH, Liu D, Sarparanta MP, Airaksinen AJ, Salonen JJ, Hirvonen JT and Santos HA: Copper-free azide-alkyne cycloaddition of targeting peptides to porous silicon nanoparticles for intracellular drug uptake. Biomaterials 35: 1257-1266, 2014

35. Sha H, Zou Z, Xin K, Bian X, Cai X, Lu W, Chen J, Chen G, Huang L, Blair AM, et al: Tumor-penetrating peptide fused EGFR single-domain antibody enhances cancer drug penetration into 3D multicellular spheroids and facilitates effective gastric cancer therapy. J Control Release 200: 188-200, 2015.

36. Li M, Tang Z, Zhang D, Sun H, Liu H, Zhang Y, Zhang Y and Chen X: Doxorubicin-loaded polysaccharide nanoparticles suppress the growth of murine colorectal carcinoma and inhibit the metastasis of murine mammary carcinoma in rodent models Biomaterials 51: 161-172, 2015.

37. Peng ZH and Kopeček J: Synthesis and activity of tumor-homing peptide iRGD and histone deacetylase inhibitor valproic acid conjugate. Bioorg Med Chem Lett 24: 1928-1933, 2014.

38. Zhang Q, Zhang Y, Li K, Wang H, Li H and Zheng J: A novel strategy to improve the therapeutic efficacy of gemcitabine for non-small cell lung cancer by the tumor-penetrating peptide iRGD. PLoS One 10: e0129865, 2015.
39. Lao X, Li B, Liu M, Chen J, Gao X and Zheng H: Increased antitumor activity of tumor-specific peptide modified thymopentin. Biochimie 107: 277-285, 2014.

40. Chen R, Braun GB, Luo X, Sugahara KN, Teesalu T and Ruoslahti E: Application of a proapoptotic peptide to intratumorally spreading cancer therapy. Cancer Res 73: 1352-1361, 2013.

41. Mao X, Liu J, Gong Z, Zhang H, Lu Y, Zou H, Yu Y, Chen Y, Sun Z, Li W, et al: iRGD-conjugated DSPE-PEG2000 nanomicelles for targeted delivery of salinomycin for treatment of both liver cancer cells and cancer stem cells. Nanomedicine (Lond) 10: 2677-2695, 2015.

42. Sugahara KN, Braun GB, de Mendoza TH, Kotamraju VR, French RP, Lowy AM, Teesalu T and Ruoslahti E: Tumor-penetrating iRGD peptide inhibits metastasis. Mol Cancer Ther 14: 120-128, 2015.

43. Hamilton AM, Aidoudi-Ahmed S, Sharma S, Kotamraju VR, Foster PJ, Sugahara KN, Ruoslahti E and Rutt BK: Nanoparticles coated with the tumor-penetrating peptide iRGD reduce experimental breast cancer metastasis in the brain. J Mol Med (Berl) 93 991-1001, 2015.

44. Ni D, Ding H, Liu S, Yue H, Bao Y, Wang Z, Su Z, Wei W and Ma G: Superior intratumoral penetration of paclitaxel nanodots strengthens tumor restriction and metastasis prevention. Small 11: 2518-2526, 2015.

45. Cao Y: Angiogenesis: What can it offer for future medicine? Exp Cell Res 316: 1304-1308, 2010.

46. Hoffman JA, Giraudo E, Singh M, Zhang L, Inoue M, Porkka K, Hanahan D and Ruoslahti E: Progressive vascular changes in a transgenic mouse model of squamous cell carcinoma. Cancer Cell 4: 383-391, 2003.

47. Singh RK, Bucana CD, Gutman M, Fan D, Wilson MR and Fidler IJ: Organ site-dependent expression of basic fibroblast growth factor in human renal cell carcinoma cells. Am J Pathol 145: 365-374, 1994.

48. Li ZJ, Wu WK, Ng SS, Yu L, Li HT, Wong CC, Wu YC, Zhang L, Ren SX, Sun XG, et al: A novel peptide specifically targeting the vasculature of orthotopic colorectal cancer for imaging detection and drug delivery. J Control Release 148: 292-302, 2010.

49. Ariztia EV, Lee CJ, Gogoi R and Fishman DA: The tumor microenvironment: Key to early detection. Crit Rev Clin Lab Sci 43 $393-425,2006$

50. Lahdenranta J, Sidman RL, Pasqualini R and Arap W: Treatment of hypoxia-induced retinopathy with targeted proapoptotic peptidomimetic in a mouse model of disease. FASEB J 21:3272-3278, 2007.

51. Buehler A, van Zandvoort MA, Stelt BJ, Hackeng TM, Schrans-Stassen BH, Bennaghmouch A, Hofstra L, Cleutjens JP, Duijvestijn A, Smeets MB, et al: cNGR: A novel homing sequence for CD13/APN targeted molecular imaging of murine cardiac angiogenesis in vivo. Arterioscler Thromb Vasc Biol 26: 2681-2687, 2006 